\title{
Isoélectrofocalisation des lactosérums de fromages de mélange brebis-vache sur gel de polyacrylamide - Application à la recherche et au dosage du lait de vache dans les fromages de brebis
}

\section{S. Rispoli et R. Saugues}

Laboratoire interrégional de la Direction générale de la consommation, de la concurrence et de la répression des fraudes, 2, rue Saint-Pierre, 34000 Montpellier, France

(reçu le 19 mai 1988, accepté le 12 décembre 1988)

Résumé - Des fromages de type fermier ont été fabriqués à partir de mélanges de lait brebisvache; ils ont été affinés en cave pendant un temps variant de 1 à 4 mois. Les lactosérums de ces fromages ont été extraits et analysés par isoélectrofocalisation (IEF) sur gel de polyacrylamide (gradient 4-6). Des bandes spécifiques du lait de vache et du lait de brebis étaient repérées. A partir de l'analyse densitométrique des IEF, nous avons calculé les rapports de densité de coloration des bandes V/B; V et B étant des bandes choisies pour caractériser respectivement le lait de vache et le lait de brebis. L'expression de ce rapport en fonction du pourcentage de lait de vache dans le mélange a permis d'établir un abaque. Cet abaque a été utilisé pour évaluer la quantité de lait de vache entrant dans la fabrication d'un fromage de mélange brebis-vache.

isoélectrofocalisation — lactosérum — fromages au lait de mélange

Summary - Isoelectric focusing of wheys of mixture milk cheeses ewe-cow in polyacrylamide gel. Application to evaluation of cow's milk in ewe's cheeses. Farm type cheeses made from a mixture of ewe's and cow's milk were ripened in a cellar over a period of 1 to 4 months. The whey from these cheeses was extracted and analysed by isoelectrofocusing (IEF) on polyacrylamide gel (gradient 4-6). Specific bands of cow's and ewe's milk were identified. Densitometric analysis of the IEF patterns were used to calculate the density coloration ratios of the C/E bands; $C$ and $E$ corresponding bands chosen to characterize cow and ewe's milk respectively. From the expression of this ratio, according to the percentage of cow's milk in the mixture, an abacus was established. This abacus was used to assess the quantity of cow's milk added to ewe's milk during the manufacture of cheeses.

isoelectrofocusing - wheys - mixture milk cheeses 


\section{Introduction}

Les fromages de brebis ou de chèvre doivent être fabriqués exclusivement avec du lait de brebis ou de chèvre. Le lait de vache étant d'un coût inférieur à celui des laits de brebis ou de chèvre, l'ajout de lait de vache lors de la fabrication de ces fromages présente un intérêt économique et constitue une fraude.

La mise en évidence de cette fraude ainsi que l'analyse des fromages au lait de mélange nécessitent des méthodes permettant de détecter les mélanges de lait de différentes espèces. Les méthodes dont nous disposons sont: les techniques chromatographiques basées sur la caractérisation de la matière grasse ou des acides aminés, les techniques immunologiques et les techniques électrophorétiques (Ramos et Juarez, 1984).

En France, actuellement, les méthodes officielles de mise en évidence de ces adultérations ne concernent que les laits et les fromages de chèvre et sont de 2 types : méthodes immunologiques (inhibition de l'hémagglutination - immunodiffusion radiale) et électrophorétiques.

Des 2 techniques immunologiques officielles, l'immunodiffusion radiale est la plus utilisée; elle donne des résultats satisfaisants, à condition que le lait n'ait pas subi un traitement thermique trop important. A cette limite, dans le cas des fromages, s'ajoute l'imprécision liée au facteur de correction tenant compte de la matière sèche du fromage (méthodes officielles).

La technique électrophorétique, basée sur la différence de mobilité de la caséine $\alpha s_{1}$ de vache par rapport aux caséines $\alpha s$ de chèvre, présente une limite d'utilisation pour les fromages affinés; la protéolyse résultant de l'affinage fait apparaître de nombreuses bandes sur le diagramme électrophorétique, le rendant ainsi difficile à interpréter (Pierre et Portmann, 1970).

Compte tenu de leurs limites, ces méthodes mises au point pour analyser les laits et fromages de chèvre ne sont pas applicables dans tous les cas à l'analyse des fromages de brebis. Le facteur de correction qui permettrait d'adapter l'immunodiffusion radiale avec une précision satisfaisante n'a pas été établi en raison des techniques de fabrication très diverses des fromages de brebis. D'autre part, les fromages de brebis sont le plus souvent commercialisés après un long affinage, ce qui rend difficile voire impossible leur analyse par la méthode électrophorétique officielle.

II s'avère donc nécessaire de mettre au point une méthode d'analyse des fromages de brebis utilisable dans tous les cas; notre étude s'inscrit dans cette optique. Nous nous sommes tournées vers l'isoélectrofocalisation (IEF). Cette méthode de plus en plus pratiquée permet de séparer plus finement les protéines (Righetti et Drysdale, 1971). L'IEF a été utilisée par plusieurs auteurs pour identifier les caséines bovines (Josephson, 1973; Trieu-Cuot et Gripon, 1981; Addeo et al., 1983) mais également pour analyser les mélanges de lait de différentes espèces. En 1982, Krause et al. proposent une méthode de détection du lait de vache dans les laits de chèvre et de brebis en utilisant la caséine gamma $(\gamma)$ comme indicateur.

Addeo et al. (1983) mettent en évidence le lait de vache dans la mozarella de bufflonne par l'étude des caséines bêta (B); en 1984, les mêmes auteurs s'intéressent aux paracaséines kappa $(\kappa)$ de vache et de brebis pour rechercher et doser le lait de vache dans le fromage de brebis pecorino. A partir des protéines de lactosérum, on peut également identifier les laits de différentes espèces (Ruiz-Mar- 
tinez et Santillana-Lopez, 1986); cependant, elles ont été moins étudiées que les caséines dans l'analyse des mélanges. C'est pourquoi nous avons choisi de nous intéresser, dans un premier temps, aux lactosérums.

Dans le présent travail, nous avons fabriqué des fromages témoins de type fermier à partir de mélanges de lait brebis-vache. Après un affinage variant de 1 à 4 mois, nous avons extrait de ces fromages les phases aqueuses contenant les protéines sériques; les fractions obtenues ont été analysées par IEF sur gel de polyacrylamide.

\section{Matériel et Méthodes}

\section{Fabrication des fromages}

Des fromages "type fermier» sont fabriqués à partir de mélanges de lait de vache et de brebis. Après réalisation des mélanges, le lait est porté à une température de $32^{\circ} \mathrm{C}$. L'emprésurage est effectué à raison de $2 \mathrm{ml}$ d'extrait de présure pour 10 litres de lait. La température est maintenue à $32{ }^{\circ} \mathrm{C}$ au bain-marie. Au bout de $2 \mathrm{~h}$, il est procédé au rompage du caillé. Après un repos de $45 \mathrm{~min}$, le caillé est égoutté puis mis en moule. Les fromages sont retournés et salés à sec par frottement. L'affinage se poursuit en cave.

\section{Première fabrication réalisée en 1986}

Les laits mis en œuvre pour cette première série d'expériences sont : le pur lait de brebis, les mélanges de lait brebis-vache contenant 5 , 25,50 ou $80 \%$ de lait de vache et le pur lait de vache. A partir de chacun de ces mélanges, nous réalisons 4 fromages dont le temps d'affinage sera de 1, 2, 3 et 4 mois. Les quantités de lait entrant en fabrication ont été calculées en tenant compte des rendements fromagers des laits de vache et de brebis.

\section{Deuxième fabrication réalisée en 1987}

Pour cette seconde série, la gamme de mélanges est élargie; les laits mis en œuvre contiennent respectivement $5,7,10,15,20$, $25,30,40,50,60,65,70,80,85$ et $95 \%$ de lait de vache. Des témoins pur brebis et pur vache sont également réalisés. Tous ces fromages sont affinés en cave pendant 3 mois. Dans tous les cas, dès réception, les fromages sont coupés en morceaux d'environ 15 grammes et sont conservés par congélation jusqu'à utilisation.

\section{Préparation des échantillons pour l'iso- électrofocalisation (IEF)}

Cinq grammes de fromage sont broyés dans $10 \mathrm{ml}$ d'eau distillée. Le mélange est ajusté à $\mathrm{pH} 4,6$ puis centrifugé pendant 15 min à 8000 tours/minute. Le surnageant contenant les protéines du lactosérum est récupéré : les traces de matière grasse sont éliminées par un traitement au chloroforme à raison de $1 \mathrm{ml}$ de lactosérum pour $2 \mathrm{ml}$ de chloroforme.

\section{Isoélectrofocalisation (IEF)}

L'IEF est réalisée sur un gel de polyacrylamide de $0,8 \mathrm{~mm}$ d'épaisseur, maintenu sur une plaque de $100 \times 125 \mathrm{~mm}$. Ce gel contient $5 \%$ d'acrylamide, $0,15 \%$ de Bis acrylamide, $6,6 \mathrm{M}$ d'urée (Biorad) et 2,2\% d'ampholytes (Biolytes $4-6,3-10,3-5$, aux concentrations respectives de $70 \%, 15 \%, 15 \%$ ).

Les solutions électrolytiques sont respectivement $\mathrm{H}_{3} \mathrm{PO}_{4} 1 \mathrm{~N}$ pour l'anode et $\mathrm{NaOH} 1 \mathrm{~N}$ pour la cathode. Les dépôts d'échantillons sont effectués par l'intermédiaire d'un carré $(6 \mathrm{~mm}$ x $6 \mathrm{~mm}$ ) de papier whatman $n^{\circ} 3$ immergé dans la solution protéique à étudier. L'IEF est effectuée à $4^{\circ} \mathrm{C}$ et à une puissance constante de 6 watts (générateur Biorad $1420 \mathrm{~B}$, cellule IEF 1415 Biorad). On ne réalise pas de préfocalisation. Après $45 \mathrm{~min}$ de passage du courant, les papiers servant aux dépôts des échantillons sont retirés et l'IEF est poursuivie pendant $2 \mathrm{~h}$. Le gel est ensuite coloré selon la méthode de Blakesley et Boezi (1977). L'analyse des résultats se fait par lecture densitométrique des bandes protéiques obtenues, à l'aide d'un densitomètre TLC Scanner II Camag.

Les gels peuvent être conservés de la façon suivante : après les avoir immergés dans une solution aqueuse à $5 \%(V / V)$ de glycérol, ils sont recouverts d'une feuille de cellophane puis séchés. 


\section{Résultats}

\section{Etude des fromages témoins}

Poids et aspect des fromages obtenus selon le temps d'affinage

Le poids diminue au cours de l'affinage, on remarque qu'en général cette perte de poids devient moins importante entre 3 et 4 mois (Tableau I). Après un mois de fabrication, la pâte des fromages est souple et blanche; à partir de 3 mois d'affinage, elle est plus ferme et plus compacte, sa couleur est jaunâtre.

Isoélectrofocalisation des fractions contenant les protéines du sérum

1) Etude des profils d'IEF. Les diagrammes d'IEF sont représentés sur la Figure 1 pour les fromages de la première fabrication à 1 mois et à 4 mois d'affinage, et sur les Figures 2 et 3 pour les fromages de la deuxième fabrication.

Pour l'échantillon «pur brebis», parmi les nombreuses bandes de focalisation, 2 sont facilement repérables dans tous les cas : $B_{1}, B_{2}$. La bande $B_{1}$ étant parfois mal séparée d'une bande mineure, nous choisissons $\mathrm{B}_{2}$ pour caractériser le lait de brebis.

Pour l'échantillon "pur vache», les bandes importantes sont regroupées en 2 zones essentielles de focalisation situées vers des $\mathrm{pH}$ plus acides que ceux observés pour les bandes de "brebis". La zone supérieure est constituée de 2 ou 3 bandes plus ou moins séparées; la zone inférieure est la bande $V$ toujours nette et dense.

Dans les mélanges, les bandes de migration provenant du lait de vache sont identifiées et séparées de celles provenant du lait de brebis. La bande la plus dense et la plus nette de vache $V$ est visible même dans les mélanges contenant $5 \%$ de lait de vache. Cette bande V est donc choisie pour caractériser le lait de vache.

Pour la première fabrication, nous retrouvons le même profil d'IEF que l'affinage soit de 1 mois ou de 4 mois. Les profils d'IEF sont similaires pour les deux fabrications réalisées à un an d'intervalle.

Tableau I. Poids des fromages de la $1^{\text {re }}$ fabrication selon le temps d'affinage (en $\mathrm{g}$ ).

\begin{tabular}{lcccc}
\hline Type de fromage & \multicolumn{3}{c}{ Temps d'affinage } \\
\cline { 2 - 5 } & 1 mois & 2 mois & 3 mois & 4 mois \\
\hline Pur brebis & 326 & 318 & 260 & 253 \\
$5 \%$ vache & 333 & 291 & 248 & 227 \\
$25 \%$ vache & 338 & 308 & 277 & 257 \\
$50 \%$ vache & 380 & 323 & 290 & 267 \\
$80 \%$ vache & 442 & 397 & 335 & 305 \\
Pur vache & 521 & 450 & 440 & 330 \\
\hline
\end{tabular}


2) Analyse densitométrique des IEF. La Figure 4 représente les diagrammes des lectures densitométriques pour les échantillons "pur brebis", "pur vache», " $25 \%$ vache", provenant des fromages de la première fabrication à 4 mois d'affinage.

La figure 5 représente les diagrammes des lectures densitométriques pour les échantillons "pur brebis", "pur vache",
« $50 \%$ vache» et $~ « 5 \%$ vache" provenant des fromages de la deuxième fabrication. Dans tous les cas, les bandes choisies pour caractériser les espèces "vache" et "brebis" (bandes $V$ et $B_{2}$ ) donnent à la lecture densitométrique des pics séparés.

3) Calcul des rapports $v / b_{2}$. Etablissement de courbes. Les surfaces des pics obtenus précédemment sont calculées

BANDE $\mathrm{V} \longrightarrow$
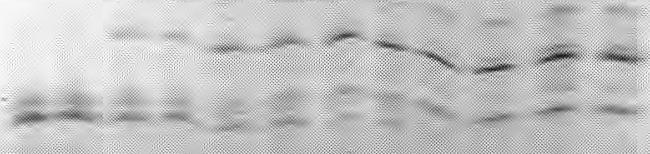

$$
\begin{array}{llllllllllll}
12 & 11 & 10 & 9 & 8 & 7 & 6 & 5 & 4 & 3 & 2 & 1
\end{array}
$$

Fig. 1. Profils d'IEF des lactosérums des fromages à 1 mois et à 4 mois d'affinage, $1^{\text {re fabrication }}$ (de droite à gauche) : 1) pur brebis 1 mois; 2) pur brebis 4 mois; 3) $5 \%$ vache 1 mois; 4) $5 \%$ vache 4 mois; 5) $25 \%$ vache 1 mois; 6) $25 \%$ vache 4 mois; 7) $50 \%$ vache 1 mois; 8 ) $50 \%$ vache 4 mois; 9) $80 \%$ vache 1 mois; 10) $80 \%$ vache 4 mois; 11) pur vache 1 mois; 12) pur vache 4 mois.

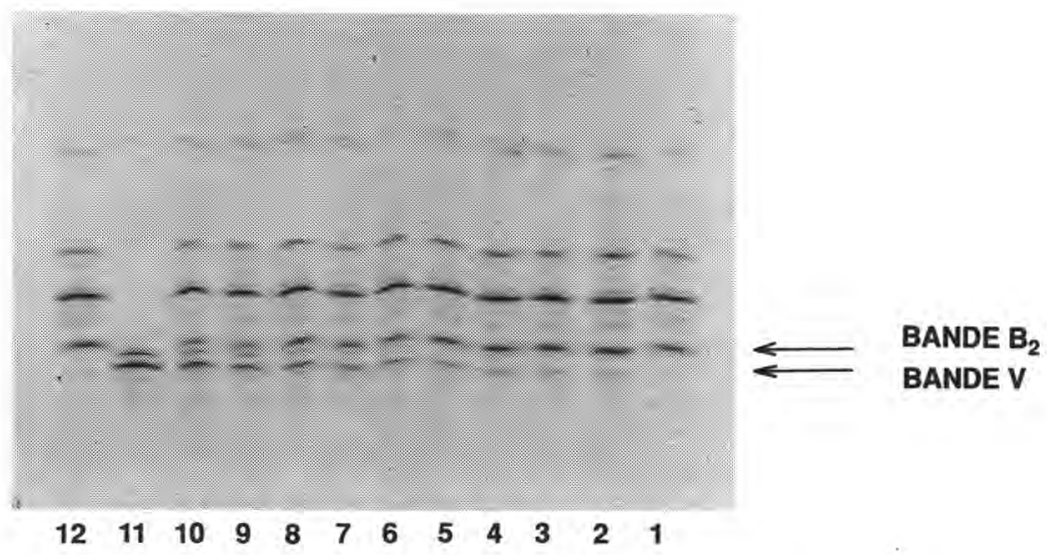

Fig. 2. Profils d'IEF des lactosérums des fromages de la $2^{\theta}$ fabrication (de droite à gauche) : 1) pur brebis; 2) $5 \%$ vache; 3) $7 \%$ vache; 4) $10 \%$ vache; 5) $15 \%$ vache; 6) $20 \%$ vache; 7) $25 \%$ vache; 8) $30 \%$ vache; 9) $40 \%$ vache; 10 ) $50 \%$ vache; 11) pur vache; 12) pur brebis. 


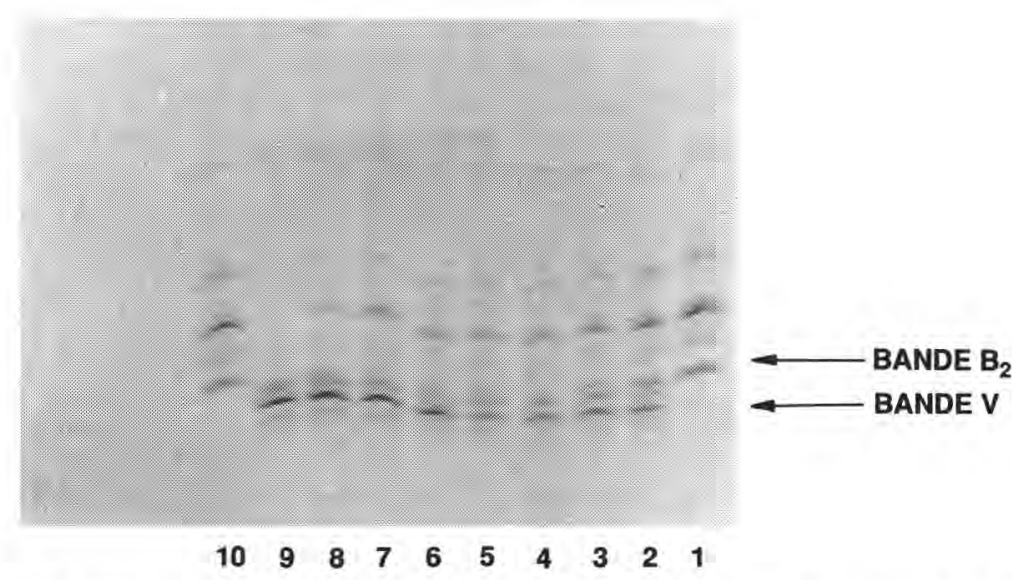

Fig. 3. Profils d'IEF des lactosérums des fromages de la $2^{e}$ fabrication : 1) pur brebis; 2) $50 \%$ vache; 3) $60 \%$ vache; 4) $65 \%$ vache; 5) $70 \%$ vache; 6) $80 \%$ vache; 7) $85 \%$ vache; 8) $95 \%$ vache; 9) pur vache; 10) pur brebis.

par l'intégrateur; $v$ et $b_{2}$ étant les surfaces des pics correspondant aux bandes $\mathrm{V}$ et $B 2$ nous exprimons le rapport $v / b_{2}$ en fonction du pourcentage de lait de vache. A partir de $10 \mathrm{IEF}$, réalisées pour l'une et l'autre des fabrications comprenant chacune toute la gamme de mélanges étudiée, des valeurs moyennes des rapports $\mathrm{v} / \mathrm{b}_{2}$ sont calculées. Ces valeurs moyennes, ainsi que les valeurs extrêmes obtenues, figurent dans le Tableau II. Les courbes moyennes obtenues sont représentées sur les Figures 6 et 7 .

Application à l'évaluation du pourcentage de lait de vache dans des fromages de mélange en utilisant une courbe établie à partir des fromages témoins

Nous avons déterminé le pourcentage de lait de vache dans les quatre fromages suivants : 2 fromages mi-brebis, c'est-àdire fabriqués à partir d'un mélange contenant $50 \%$ de lait de vache; 2 fromages de mélange brebis-vache fabriqués avec un mélange contenant $65 \%$ de lait de vache.
Pour cela, nous avons effectué sur la même plaque I'IEF des échantillons de ces fromages et de fromages de la gamme témoin (Fig. 8). Les rapports $\mathrm{v} / \mathrm{b}_{2}$ ont été calculés pour chaque échantillon déposé sur la plaque. A partir de la courbe témoin, nous évaluons le pourcentage de lait de vache de chacun des fromages étudiés.

Les résultats suivants sont obtenus : mi-brebis $\mathrm{n}^{\circ} 1: 50 \% \mathrm{~V}$, mi-brebis $n^{\circ} 2: 55 \% \mathrm{~V}$, $65 \% n^{\circ} 1: 70 \%$, $65 \% n^{\circ} 2: 63 \%$ V.

\section{Discussion}

La première fabrication a permis de mettre en évidence, par IEF des fractions contenant les protéines de lactosérum, des bandes spécifiques du lait de vache et du lait de brebis. Les bandes de migration choisies pour caractériser les deux espèces de lait sont nettement repérées et séparées, quel que soit le temps d'affinage $(1,2,3$ ou 4 mois); ceci constitue 


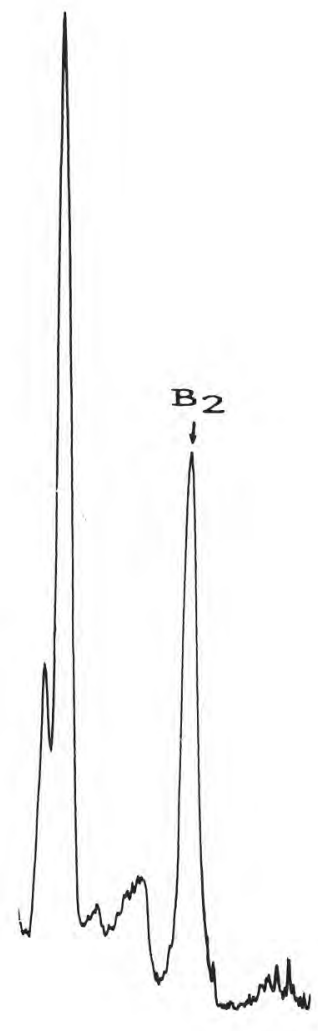

PUR BREBIS

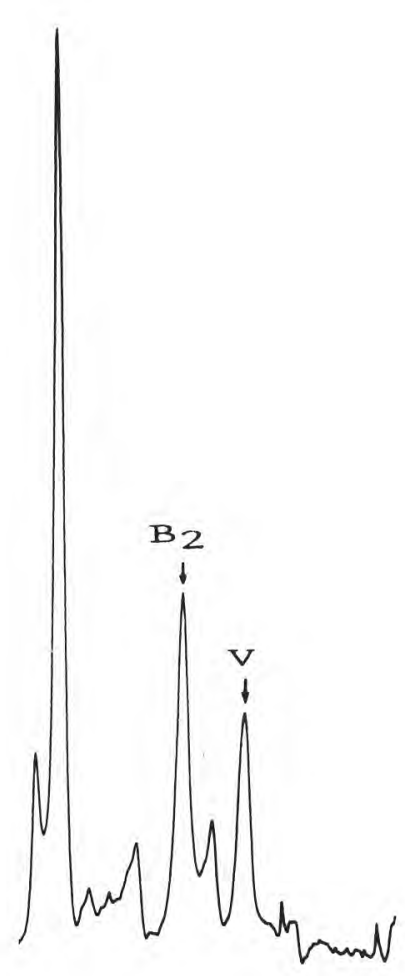

$25 \%$ VACHE

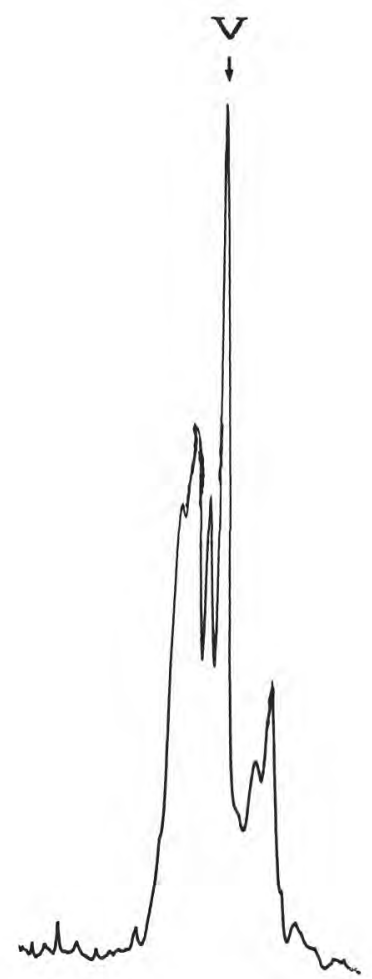

PUR VACHE

Fig. 4. Lectures densitométriques (à $600 \mathrm{~nm}$ en transmission) des bandes d'IEF des échantillons : pur brebis; $25 \%$ vache; pur vache à 4 mois d'affinage (1re fabrication).

déjà un avantage par rapport à l'électrophorèse des caséines qui, pour ces mêmes fromages, a donné des résultats difficilement exploitables pour un affinage supérieur à 1 mois.

Les profils d'IEF étant similaires quel que soit le temps d'affinage, pour la seconde fabrication nous avons élargi la gamme de mélange et fixé le temps d'affinage à 3 mois.

A partir des analyses densitométriques des bandes choisies, nous représentons $v / b_{2}$ en fonction du pourcentage de lait de vache. Lorsque les échantillons d'une gamme de mélange (soit de $5 \%$ à $50 \%$ ou soit de $50 \%$ à $95 \%$ ) sont analysés sur une même plaque, les courbes obtenues dans les 2 cas sont croissantes et régulières; elles ont toujours cette même allure pour des IEF successives réalisées dans les mêmes conditions. Cependant, pour un même échantillon d'un mélange donné, analysé sur des plaques différentes, nous avons observé une certaine variabilité du rapport $v / b_{2}$ qui peut être due à des fac- 


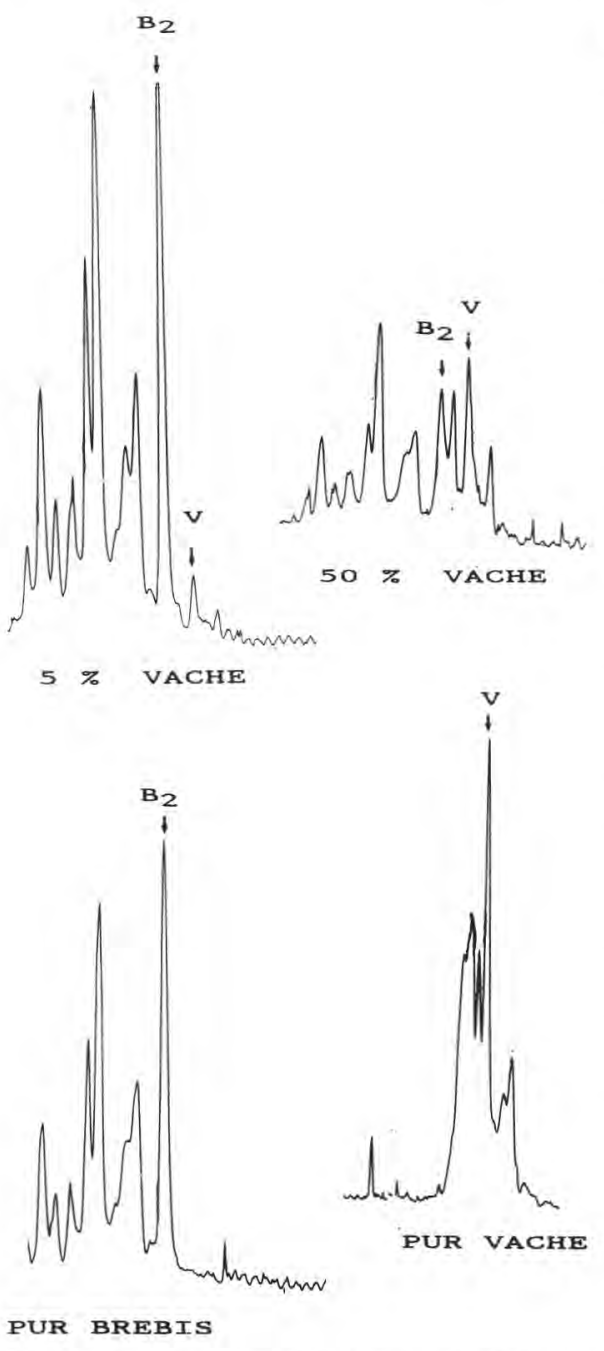

Fig. 5. Lectures densitométriques (à $600 \mathrm{~nm}$ en transmission) des bandes d'IEF pour les échantillons : pur brebis; pur vache; $5 \%$ vache : $50 \%$ vache ( $2^{\circ}$ fabrication).

teurs inhérents à la technique d'IEF (homogénéité du gel, formation du gradient, coloration des protéines). Dans le Tableau 2 figurent les valeurs moyennes des rapports $v / b_{2}$, ces moyennes ont été calculées à partir d'un nombre d'IEF ne permettant pas une analyse statistique, aussi nous avons choisi d'y adjoindre les valeurs extrêmes obtenues pour rendre compte des écarts. Nous avons constaté que cette variabilité était sans conséquence sur l'évaluation des pourcentages de lait de vache dans un fromage quelconque à condition de déposer sur la même plaque les échantillons des fromages témoins avec celui du fromage à analyser. En effet, lorsque nous avons étudié les fromages mi-brebis et $65 \%$ vache, nous avons réalisé des IEF successives, chacune d'elles comportant les échantillons de la gamme témoin et les échantillons à analyser. De cette manière, nous avons obtenu d'une plaque à l'autre, des valeurs concordantes pour le pourcentage de lait de vache malgré la variabilité des rapports $\mathrm{v} / \mathrm{b}_{2}$.

Ce travail, limité d'une part à l'étude des fractions contenant les protéines sériques et d'autre part à un seul mode de fabrication (fromage fermier au lait cru), doit être considéré comme une approche dans la mise au point d'une méthode d'analyse des fromages au lait de mélange. Etant donné que les protéines du lactosérum peuvent subir des modifications de structure plus ou moins importantes selon la nature du traitement thermique (Doi et al., 1983; Donovan et Mulvihill, 1983; Valdicelli et al., 1986; de Wit et Klarenbeek, 1984), il sera nécessaire d'étudier le profil d'IEF de fromages fabriqués à partir de lait chauffé.

Pour construire une courbe témoin permettant, quel que soit le type de fromage, d'obtenir directement le pourcentage de lait de vache sans avoir à utiliser de facteur de correction - comme c'est le cas pour l'immunodiffusion radiale - des compléments devront être apportés à cette technique : elle sera appliquée à des fromages issus de divers modes de fabrication, les bandes $V$ et $B_{2}$ seront identi- 
Tableau II. Valeurs moyennes et valeurs extrêmes de v/b

\begin{tabular}{|c|c|c|c|c|c|c|}
\hline \multirow{2}{*}{$\begin{array}{l}\% \text { du lait de vache } \\
\text { dans le mélange }\end{array}$} & \multicolumn{2}{|c|}{ valeurs moyennes } & \multicolumn{2}{|c|}{ valeurs minimales } & \multicolumn{2}{|c|}{ valeurs maximales } \\
\hline & 1986 & 1987 & 1986 & 1987 & 1986 & 1987 \\
\hline 5 & 0,19 & 0,10 & 0,10 & 0,08 & 0,25 & 0,13 \\
\hline 7 & & 0,15 & & 0,13 & & 0,18 \\
\hline 10 & & 0,18 & & 0,16 & & 0,23 \\
\hline 15 & & 0,30 & & 0,28 & & 0,34 \\
\hline 20 & & 0,35 & & 0,39 & & 0,32 \\
\hline 25 & 0,66 & 0,45 & 0,64 & 0,38 & 0,75 & 0,50 \\
\hline 30 & & 0,57 & & 0,53 & & 0,62 \\
\hline 40 & & 0,68 & & 0,65 & & 0,78 \\
\hline 50 & 1,30 & 1,10 & 1,02 & 0,90 & 1,40 & 1,20 \\
\hline 60 & & 1,30 & & 1,24 & & 1,45 \\
\hline 65 & & 1,66 & & 1,55 & & 1,70 \\
\hline 70 & & 2,25 & & 1,90 & & 2,42 \\
\hline 80 & 3,35 & 3,82 & 2,9 & 3,20 & 4,20 & 4,02 \\
\hline 85 & & 5,20 & & 4,40 & & 5,70 \\
\hline 95 & & 7,20 & & 5,50 & & 8,50 \\
\hline
\end{tabular}

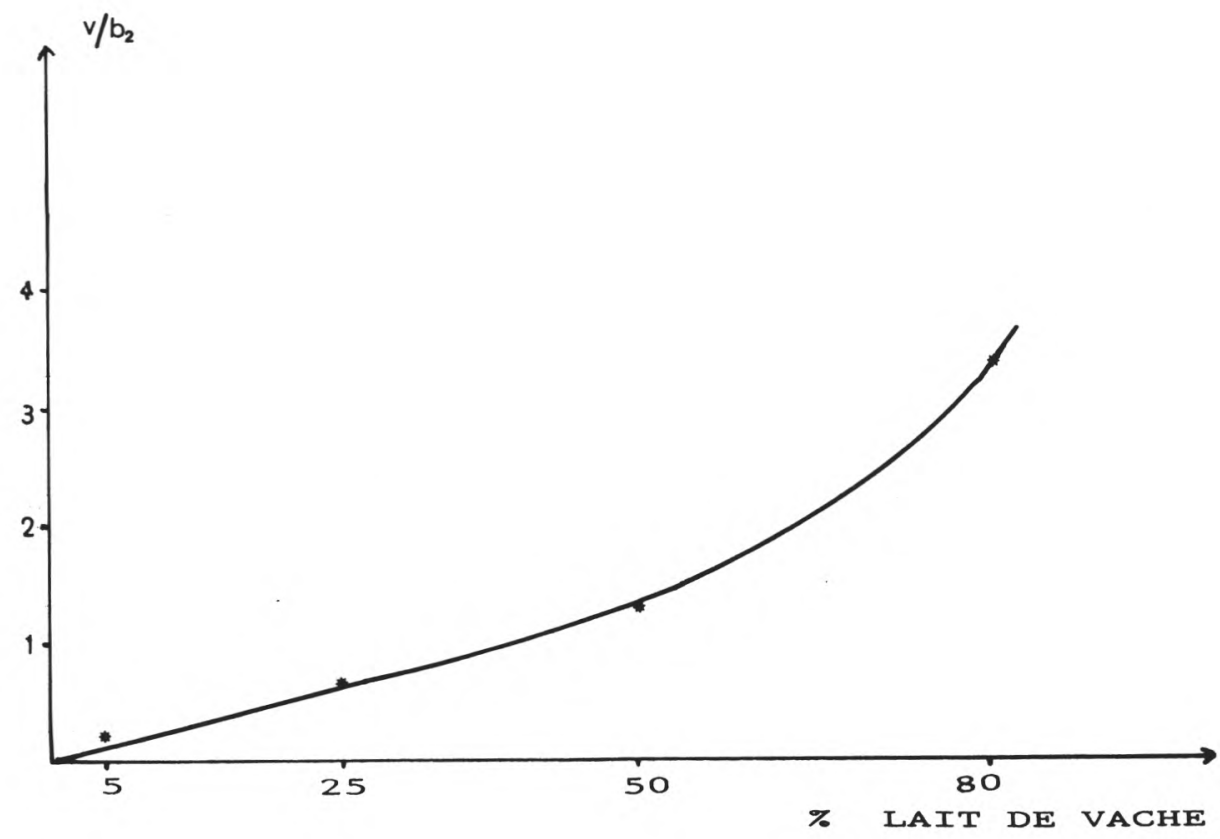

Fig. 6. Représentation graphique des valeurs moyennes des rapports $v / b_{2}$ en fonction du pourcentage de lait de vache dans le mélange (1'e fabrication). 
fiées, un élargissement de la gamme de mélange aux faibles pourcentages de lait de vache permettra d'établir le seuil de détection de la méthode.

\section{Conclusion}

Cette étude a permis de montrer qu'il est possible, à partir d'IEF de lactosérum, de
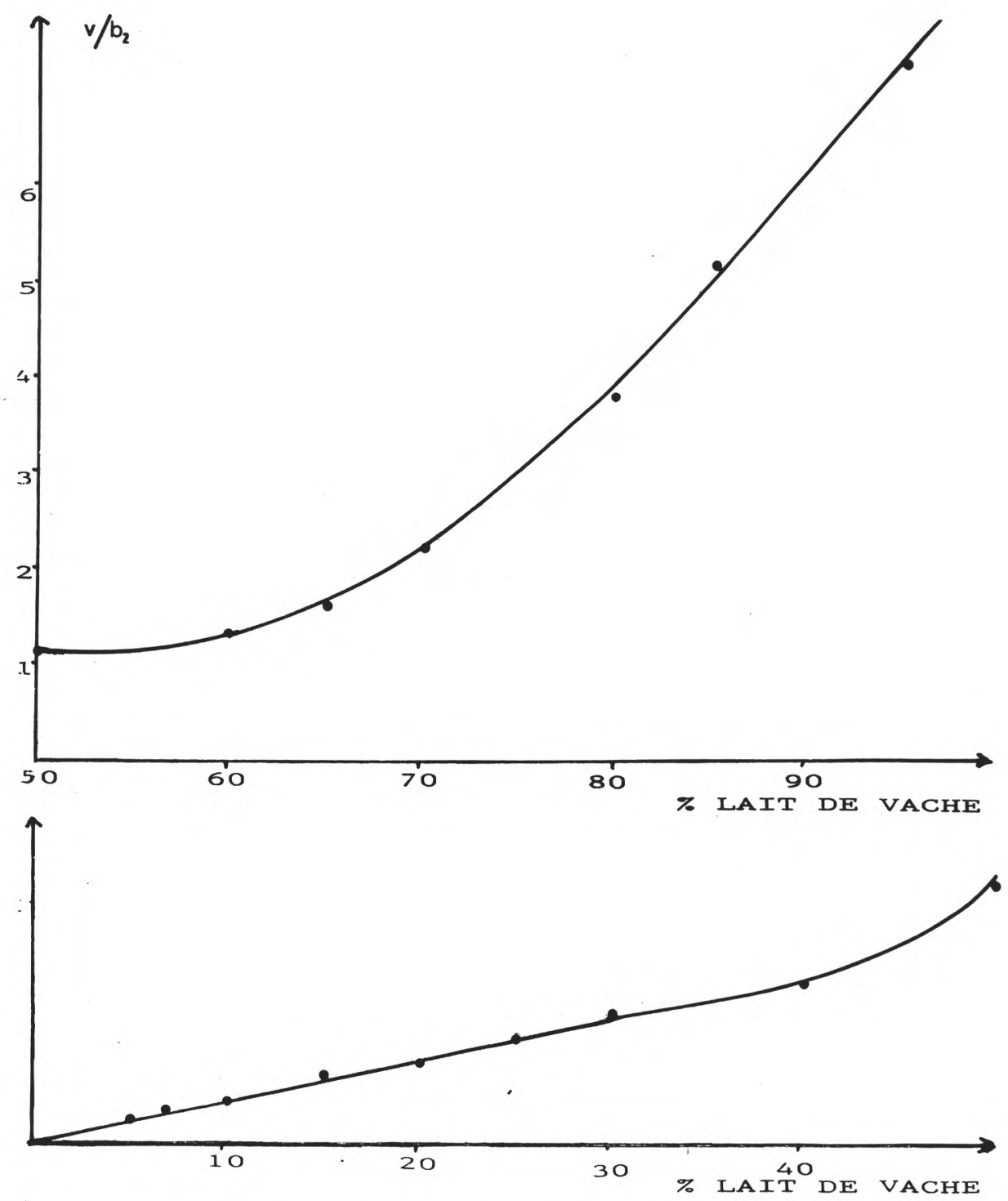

Fig. 7. Représentation graphique des valeurs moyennes des rapports $v / b_{2}$ en fonction du pourcentage de lait de vache dans le mélange ( $2^{e}$ fabrication). 


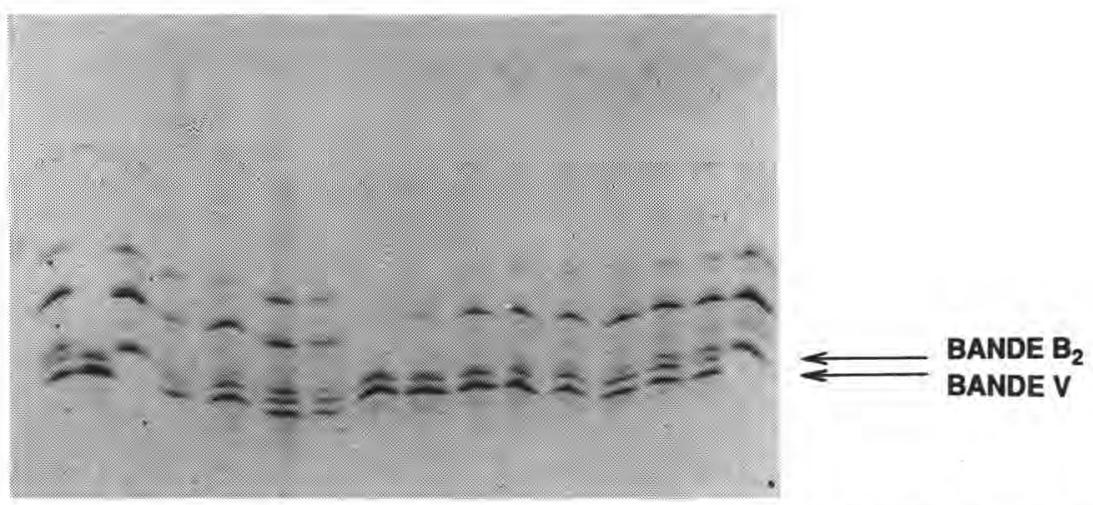

Fig. 8. Profils d'IEF des lactosérums des fromages suivants (de droite à gauche) : témoins pur brebis, $50 \%$ vache, $60 \%$ vache, $65 \%$ vache, $70 \%$ vache, $80 \%$ vache, $85 \%$ vache, $95 \%$ vache, pur vache; mi-brebis $n^{\circ} 1$; mi-brebis $n^{\circ} 2$; $65 \%$ vache $n^{\circ} 1 ; 65 \%$ vache $n^{\circ} 2$; témoins pur brebis, pur vache, $50 \%$ vache.

déceler la présence de lait de vache dans les fromages de brebis même après plusieurs mois d'affinage. A ce stade de l'étude, pour évaluer la quantité de lait de vache avec une précision satisfaisante, il est nécessaire d'effectuer I'IEF des échantillons de la gamme témoin et du fromage à analyser sur une même plaque.

La technique proposée semble donc, contrairement à l'électrophorèse des caséines, donner satisfaction pour l'analyse de fromages de brebis affinés.

Notre objectif étant la mise au point d'une méthode d'analyse des fromages de mélange applicable à tous les types de fabrication, nous envisageons d'une part de poursuivre ce travail sur les lactosérums et d'autre part de nous intéresser aux profils d'IEF des caséines et plus particulièrement des paracaséines kappa, celles-ci présentant l'avantage d'être à la fois moins thermosensibles que les protéines sériques et peu dégradées, au cours de l'affinage.

\section{Remerciements}

Nous tenons à remercier vivement MM. Simon, Bayle et Vigan, directeur départemental, inspecteur et contrôleur de la direction de la Concurrence, de la Consommation et de la Répression des fraudes de Lozère qui, par leur contribution, ont permis la fabrication des fromages témoins dans les meilleures conditions.

Toute notre gratitude va également à $M$. et $M^{m e}$ Negron, exploitants agricoles en Lozère, pour leur compétence et leur aide précieuse lors de cette fabrication.

\section{Références}

Méthodes officielles d'analyse des laits et fromages de chèvre. Arrêté du 29 mars 1978. JO du 13-4-1978 et du 1er-6-1978

Addeo F., Stingo C., Chianese L., Petrilli P., Scudiero A. \& Anelli G. (1983) Focalizzazione isoelettrica delle caseine per il riconoscimento del latte bovino nella mozarella di bufala. Latte 8, 795-803

Addeo F., Chianese L., Di Luccia A., Petrilli P., Mauriello R. \& Anelli G. (1983) Identification of bovine casein variants by gel isoelectric focusing. Milchwissenschaft $38,10,586-588$ 
Addeo F., Anelli G., Stingo C., Chianese L., Petrilli P. \& Scudiero A. (1984) Riconoscimento e dosaggio del latte bovino nel formaggio "pecorino". Mondo Latte 9, 135-142

Blakesley R.W. \& Boezi J.A. (1977) A new staining technique for proteins in polyacrylamide gels using Coomassie Brillant blue G 250 . Anal. Biochem. 82, 580-582

Doi M., Tokuyama T., Ideno S., Ibuki F., Miyanaka A. \& Kanamori M. (1983) Heat induced interaction between $\kappa$-casein and $\alpha$-lactalbumin. In : Basic Studies in Food Science. Boole Press Ltd, Dublin, 2, pp. 32-33

Donovan M. \& Mulvihill D.M. (1983) Thermal denaturation of proteins in rennet casein whey. In : Basic Studies in Food Science. Boole Press Ltd, Dublin, 3, 28-29

Josephson R.V. (1973) Isoelectric focusing of bovine milk caseins. J. Dairy Sci. 55, 15351543

Krause I., Belitz H.D. \& Kaiser K.P. (1982) Detection of cow's milk in sheep's and goat's milk and cheese by isoelectric focusing on thin layers of polyacrylamide gels containing urea. Z. Lebensm. Unters. Forsch. 174, 195-199
Pierre A. \& Portmann A. (1970) Emploi de l'électrophorèse en gel de polyacrylamide pour mettre en évidence et doser le lait de vache ajouté au lait de chèvre. Application au cas des fromages. Ann. Technol. Agric. 19, 107-130

Ramos M. \& Juarez M. (1984) Update on existing analytical methods for detecting mixtures of cow's, ewe's and goat's milk. Bull. FIL IDF document 181

Righetti P. \& Drysdale S.W. (1971) Isoelectric focusing in polyacrylamide gels. Biochim. Biophys. Acta 293, 17-28

Ruiz Martinez E. \& Santillana Lopez I. (1986) Determinación de mezclas de leche de vaca, cabra y oveja, por isoelectroenfoque en gel de poliacrilamida. Alimentaria, Abril, 55-64

Trieu-Cuot P. \& Gripon J.-C. (1981) Electrofocusing and two-dimensional electrophoresis of bovine caseins. J. Dairy Res. 48, 303-310

Valdicelli L., Neviani E. \& Emaldi G.C. (1986) Sensibilità delle sieroproteine del latte ai trattammenti termici. Latte 11, 953-958

Wit J.N. de \& Klarenbeek G. (1984) Effects of various heat treatments on structure and solubility of whey proteins. J. Dairy Sci. 67, 27012710 